\title{
Voltage-Tunable Dual-Band In(Ga)As Quantum-Ring Infrared Photodetector
}

\author{
Jong-Horng Dai, Yi-lung Lin, and Si-Chen Lee, Fellow, IEEE
}

\begin{abstract}
The ten-period In(Ga)As quantum-ring infrared photodetector (QRIP) prepared by molecular beam epitaxy is investigated. The quantum rings show narrow-sized distributions that are segregated into two groups. The QRIP demonstrates a dual-band operation with response peak shifting from the long wavelength of $9.5 \mu \mathrm{m}$ at biases less than $0.6 \mathrm{~V}$ to the middle wavelength of $6.8 \mu \mathrm{m}$ at biases larger than $0.8 \mathrm{~V}$. The maximum peak responsivity at $20 \mathrm{~K}$ is $422 \mathrm{~mA} / \mathrm{W}$ at $1.2-\mathrm{V}$ bias.
\end{abstract}

Index Terms-Dual band, infrared photodetector, quantum dots (QDs), quantum ring.

\section{INTRODUCTION}

$\mathbf{M}$ ULTICOLOR or broadband infrared (IR) photodetectors have attracted much attention in recent years due to their potential applications [1]-[6]. They include the fabrication of low-dimensional quantum structures such as quantum wells (QWs) and quantum dots (QDs) for quantum-well infrared photodetectors (QWIPs) and quantum-dot infrared photodetectors (QDIPs), respectively [7], [8]. Although QWIPs have the flexibility of bandgap engineering that can be designed to detect different wavelength ranges, their disadvantages include narrowband detection and forbidden normal incident absorption [9]. The QDIPs have solved these two problems. The growth procedure of the $\operatorname{In}(\mathrm{Ga})$ As-GaAs quantum-ring $(\mathrm{QR})$ structure is quite similar to those of the QD structure, except for an additional thin GaAs cap layer on InAs QD and a following annealing procedure for $\operatorname{In}(\mathrm{Ga})$ As $Q R$ formation. The confinement region is defined by capped GaAs thickness; hence, the transition energy between subbands is tailorable by the growth procedure [10], [11]. The device application of QRs was only in the area of semiconductor laser [12]; the possibility of applying QR structure in IR photodetector is still unexplored. In this letter, the $\mathrm{In}(\mathrm{Ga}) \mathrm{As}-\mathrm{GaAs}$ quantum-ring infrared photodetector (QRIP) is fabricated by stacking ten layers of $\operatorname{In}(\mathrm{Ga})$ As QRs separated by 30-nm GaAs spacer layer in the active region. The spectral response demonstrates that the QRIP is a voltage-tunable dual-band detector. The physical mechanism responsible for dual band is discussed.

\section{DEVICE FABRICATION}

The samples are prepared by VG $80 \mathrm{H}$ solid source molecular beam epitaxy (MBE) on semi-insulating (100) GaAs substrates. The growth rates of the InAs and GaAs layers are 0.26 monolayer (ML)/s and $1 \mu \mathrm{m} / \mathrm{h}$, respectively. The $200-\mathrm{nm}$

Manuscript received January 22, 2007; revised May 15, 2007. This work was supported by the National Science Council under Contract NSC-95-2120-M002-007.

The authors are with the Department of Electrical Engineering and Graduate Institute of Electronics Engineering, National Taiwan University, Taipei, Taiwan 10617, R.O.C. (e-mail: sclee@cc.ee.ntu.edu.tw).

Digital Object Identifier 10.1109/LPT.2007.903344

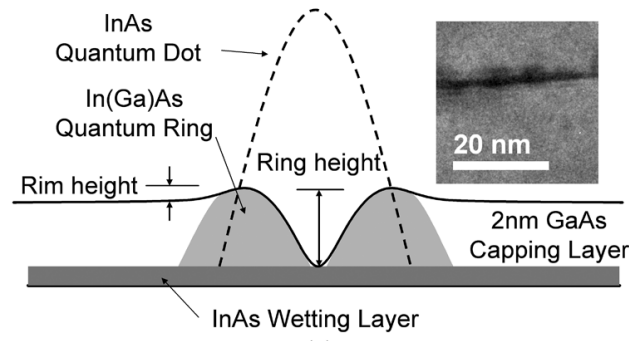

(a)

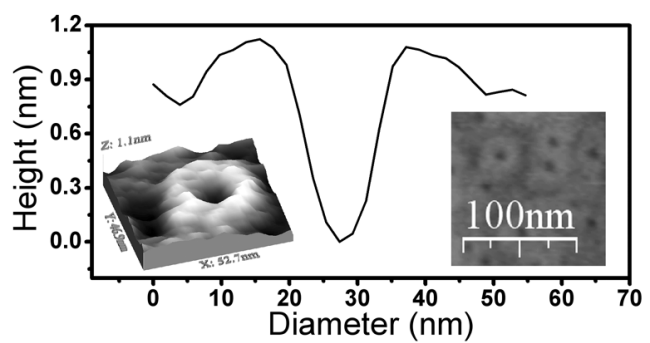

(b)

Fig. 1. (a) Sketch of the formation mechanisms of the QR. Right inset is the cross-sectional TEM image of QR. (b) Vertical profile of QR sample. Left inset is a 3-D AFM image of a single QR and right inset shows the AFM image of the sample surface in an area of $150 \times 150 \mathrm{~nm}^{2}$.

buffer layer was first grown at $600{ }^{\circ} \mathrm{C}$, the temperature of substrate was lowered to $480{ }^{\circ} \mathrm{C}$, and then the $2.5 \mathrm{ML}$ InAs QDs were grown using the Stranski-Krastanov growth mode. After closing the shutter of In cell for $20 \mathrm{~s}$, a 2-nm GaAs capping layer was deposited on top of the QDs and then the sample was annealed at the same temperature for $30 \mathrm{~s}$ to form the In(Ga)As QRs. During the annealing, the In atoms in the uncovered InAs QD move away from the QD and mix with the neighboring capped GaAs layer, as shown in Fig. 1(a). Hence, holes are formed with alloyed $\operatorname{In}(\mathrm{Ga})$ As ring around them [13]. The ring height is defined as the hole center depth. The morphology and optical properties of QRs are characterized by atomic force microscopy (AFM), transmission electron microscopy (TEM), and photoluminescence (PL) spectrum. The right inset in Fig. 1(a) is the cross-sectional TEM image of a single ring. When the sample is cut through a ring center, it looks like two cross sections of toroids adjacent to each other in the wetting layer. Fig. 1(b) shows the profile of a single QR taken from AFM. The ring height varies from 1.2 to $2.0 \mathrm{~nm}$ and average rim height is $0.3 \mathrm{~nm}$. The left inset is the $3-\mathrm{D}$ image of a single ring with an external diameter of $33 \mathrm{~nm}$ and inner diameter of $12 \mathrm{~nm}$. The QR density is about $5 \times 10^{10} \mathrm{~cm}^{-2}$. In our earlier work on the self-assembled 2.5 ML InAs QDs, the QD density was also $\sim 5 \times 10^{10} \mathrm{~cm}^{-2}$ with an average height of $\sim 3.8 \mathrm{~nm}$, but certain large QDs reached a height close to $\sim 8 \mathrm{~nm}$, suggesting a merger of two small QDs. The height of 


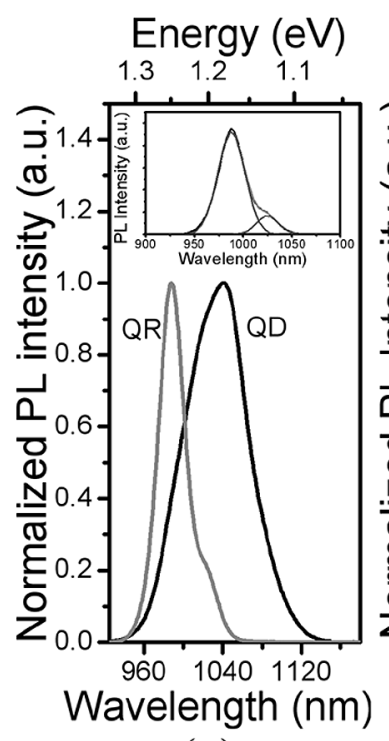

(a)

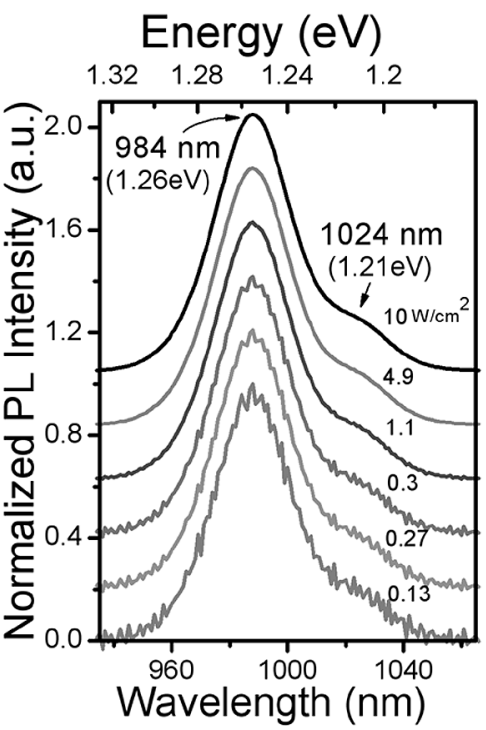

(b)
Fig. 2. (a) Normalized PL spectra of $\operatorname{In}(\mathrm{Ga}) \mathrm{As}$ QRs and QDs embedded in GaAs at measured $20 \mathrm{~K}$. Left inset shows the PL spectrum fitted by two Gaussian curves. (b) Normalized PL spectra of $\operatorname{In}(\mathrm{Ga})$ As QR sample as a function of laser power intensity.

the QD has a standard deviation of $\sim 1.6 \mathrm{~nm}$. It appears that there exist two groups of QDs causing the broadening of height distribution $(0.42,=\mathrm{sd} / \mathrm{mean})$. When the QDs are transformed to $\mathrm{In}(\mathrm{Ga}) \mathrm{As} \mathrm{QRs}$, they segregate into two apparent size groups.

The In(Ga)As QRIP was fabricated under the same growth conditions as the previous QR test sample. Sandwiched between $0.5-\mu \mathrm{m}$ top and $1-\mu \mathrm{m}$ bottom GaAs contact layers with Si doped to $1 \times 10^{18} \mathrm{~cm}^{-3}$, ten periods of QR structures with 30-nm GaAs spacer between neighboring QR layers are grown by MBE. After a two-step process, a $150 \times 150 \mu \mathrm{m}^{2}$ device is fabricated. The photoresponse of the device is measured under an $45^{\circ}$-edge coupling scheme with a close cycle cryogenic cooler and Perkin-Elmer S2000 Fourier transform IR spectroscope [14]. The absolute value of the spectral responsivity is calibrated with a standard blackbody radiation source operated at $800 \mathrm{~K}$.

\section{RESULTS AND DISCUSSION}

The 20-K PL spectrum of the QR is shown in Fig. 2(a). Compared with the $2.5 \mathrm{ML}$ InAs-GaAs QD sample, the blue shift of PL peak wavelength of the QR is attributed to the smaller ring height and larger bandgap of the alloyed $\operatorname{In}(\mathrm{Ga}) \mathrm{As}$. The full-width at half-maximum of the PL is only $49 \mathrm{meV}$, which is much narrower than that of the QD sample, that is, $\sim 88 \mathrm{meV}$. This demonstrates that the ring height distribution of QRs is not so diverse as those of QDs. The left inset shows that the QR PL spectrum can be fitted by the superposition of two Gaussian functions, one peak is at $984 \mathrm{~nm}$ and another peak is at $1024 \mathrm{~nm}$. This indicates that two groups of QRs with different sizes exist. Fig. 2(b) displays the normalized PL spectra as a function of the exciting laser power from 0.13 to $10 \mathrm{~mW} / \mathrm{cm}^{2}$. The spectra indicate that each curve consists of two peaks that are independent of the laser power. Although the AFM analysis of QDs reveals two groups of height, its PL spectrum is broadened to the level that no apparent two peaks are observed. The density of small-sized

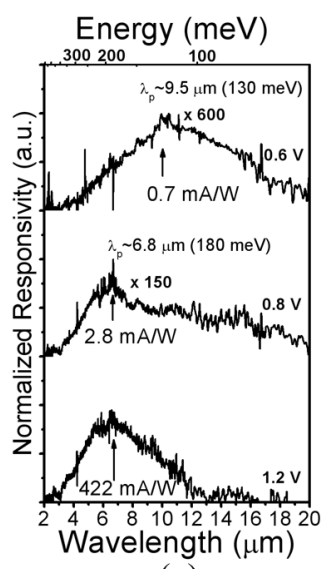

(a)

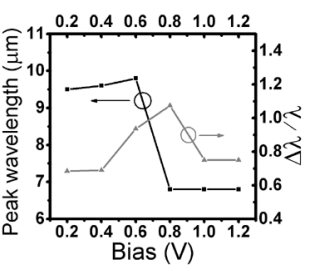

(b)

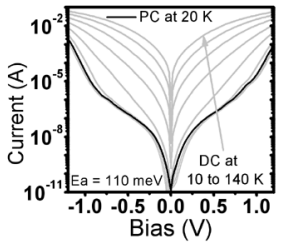

(c)
Fig. 3. (a) Spectral response of QRIP at a bias of $0.6,0.8$, and $1.2 \mathrm{~V}$ at $20 \mathrm{~K}$. (b) Peak wavelength $\lambda_{P}$ and spectral width $\left(\Delta \lambda / \lambda_{P}\right)$ measured at $20 \mathrm{~K}$ as a function of the applied voltage. (c) Dark current as a function of applied voltage of the QRIP from $10 \mathrm{~K}$ to $140 \mathrm{~K}$. The bold curve represents the photocurrent measured at $20 \mathrm{~K}$ when the QRIP is illuminated by the ambient background radiation through the cryostat window.

QRs that contribute to stronger PL peak intensity is five times larger than that of the large-sized QRs.

The normalized 20-K spectral responses of the QRIP measured at 0.6, 0.8, and 1.2 V are shown in Fig. 3(a). The peak responsivity is $422 \mathrm{~mA} / \mathrm{W}$ under a bias voltage of $1.2 \mathrm{~V}$, which is similar to that of a QDIP with the similar structure [14]. At bias lower than $0.6 \mathrm{~V}$, the response peak wavelength appears around $9.5 \mu \mathrm{m}$. When the bias reaches $0.8 \mathrm{~V}$ and beyond, the response peak wavelength shifts to $6.8 \mu \mathrm{m}$. The phenomenon originates from the two groups of QR sizes, which are consistent with the PL results, as shown in Fig. 2(a). One group of QRs is responsible for the shorter wavelength $(\sim 6.8 \mu \mathrm{m}, 180 \mathrm{meV})$ detection band at high bias; the other group of QRs rules the longer wavelength $(\sim 9.5 \mu \mathrm{m}, 130 \mathrm{meV})$ detection band at lower bias. As shown in Fig. 3(b), both the spectral width ratio $\Delta \lambda / \lambda_{P}$ and peak wavelength change dramatically at a bias around $0.7 \mathrm{~V}$. When the bias is at $0.6 \mathrm{~V}$, the spectral width ratio $\Delta \lambda / \lambda_{P}$ is 0.93 ; it increases to 1.07 at $0.8 \mathrm{~V}$. At other biases, the ratio is about 0.7 , which is much larger than the value 0.5 observed for InAs-GaAs QDIPs [15]. The results are also different from that of InGaAs QDIP [16]. The broadened bandwidth is due to the superposition of two response peaks. Fig. 3(c) shows the temperature-dependent dark current-voltage $(I-V)$ characteristics of the $\mathrm{In}(\mathrm{Ga}) \mathrm{As}$ QRIP from $10 \mathrm{~K}$ to $140 \mathrm{~K}$ at external biases of $\pm 1.2 \mathrm{~V}$. The photocurrent measures at $20 \mathrm{~K}$ with the device illuminated by the background radiation. More insight on the electron distribution in the QR structures is required to properly understand the transition mechanisms. By fitting the temperature-dependent dark currents $I_{D}$ to thermionic emission formulas $I_{D}=A * T^{2} \exp \left(-E_{a} / k T\right)$, where $A *$ is the Richardson constant, $T$ is the temperature, and $E_{a}$ is activation energy that equals $\Delta E_{C}-E_{F}, \Delta E_{C}$ is the conduction-band discontinuity and $E_{F}$ is the Fermi level, a zero-voltage activation energy can be obtained [14]. The zero-bias activation energy of the QRIP is $110 \mathrm{meV}$.

According to the result, the transition mechanisms responsible for the spectral response are established. The schematic energy band diagram for the device is shown in Fig. 4. Assuming 


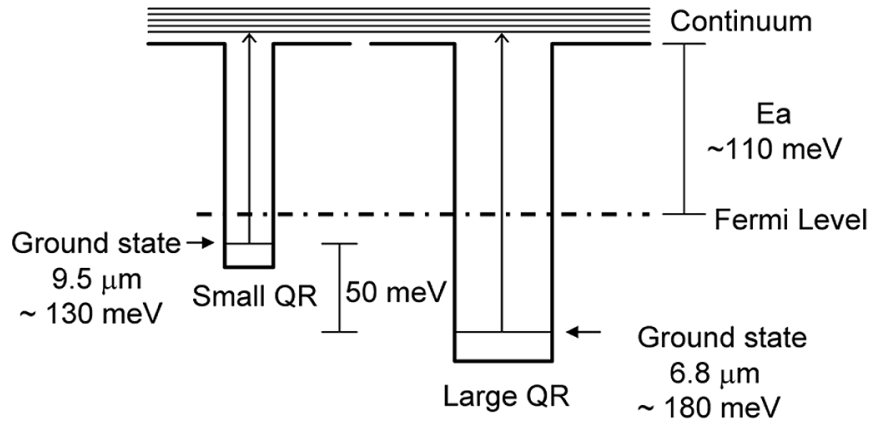

Fig. 4. Schematic energy band diagram of QRIP with two groups of QR size.

that there are two confined states in the $\mathrm{QR}$ structures, the peak at $\sim 9.5 \mu \mathrm{m}(\sim 130 \mathrm{meV})$ is attributed to ground-to-continuum transition from small QRs and the peak at $\sim 6.8 \mu \mathrm{m}(\sim 180 \mathrm{meV})$ is attributed to another ground-to-continuum transition from large QRs. At low applied bias of $0.6 \mathrm{~V}$, the ground states are all filled with electrons. From a theoretical calculation, the ground state of the small QR is higher in energy than that of the large one. Since the small QRs are denser than those of the large one, the probability of ground-to-continuum transition of small QRs is higher than those of the large QRs. Hence, the main peak response is located at $\sim 9.5 \mu \mathrm{m}(130 \mathrm{meV})$ at lower applied bias, which is contributed by small QRs. When the applied bias is increased, the band diagram will be tilted, and the quasi-Fermi level below the conduction minimum, defined as the activation energy, decreases. The zero-voltage activation energy is $110 \mathrm{meV}$, measured from Fig. 3(c). At zero bias, the ground state energy of small QRs is only $20 \mathrm{meV}$ away from the Fermi level; therefore, the Fermi level reaches very close to the ground state energy of the small QRs at higher bias than intermediate $0.7 \mathrm{~V}$. The depopulation of electrons from the ground state of small QRs diminishes the transition rate and the photoresponse of the $9.5-\mu \mathrm{m}$ peak. Then the photocurrent from large QRs become dominant at peak $\sim 6.8 \mu \mathrm{m}(180 \mathrm{meV})$. At an even higher bias, the Fermi level is relatively shifted toward the ground state of large QRs; the transition from ground state of small QRs is suppressed, and only transition in large QRs remain. The response bandwidth also decreases when the applied bias reaches 1.0 and $1.2 \mathrm{~V}$, because only a single transition peak in the large QRs exists.

\section{CONCLUSION}

A voltage-tunable dual-band QRIP has been demonstrated with peak wavelengths centered at either 9.5 or $6.8 \mu \mathrm{m}$. The QR bandgap is easily tailored to meet the specific requirement of peak response, and the growth of two groups of QR size is responsible for multicolor detection with two terminals only. It is also suitable for the broadband detection of IR with wavelength ranging from 3 to $20 \mu \mathrm{m}$. The quality of this device can be improved by changing the layer structure, that is, adding the current blocking layer [8], doping in the ring [17], [18], or stacking more layers that enhance the property of QDIPs. Finally, the QR can replace the QD in an IR photodetector that provides controllable bandgap engineering with the same performance as QD.

\section{ACKNOWLEDGMENT}

The authors would like to thank Prof. R. M. Lin of Chang Gung University for helpful discussions on PL measurement.

\section{REFERENCES}

[1] S. M. Kim and J. S. Harris, "Multicolor InGaAs quantum-dot infrared photodetectors," IEEE Photon. Technol. Lett., vol. 16, no. 11, pp. 2538-2540, Nov. 2004.

[2] A. Majumdar, K. K. Choi, J. L. Reno, and D. C. Tsui, "Voltage tunable superlattice infrared detector for mid and long-wavelength detection," Appl. Phys. Lett., vol. 86, pp. 261110-261112, 2005.

[3] S. D. Chen, Y. Y. Chen, and S. C. Lee, "High-performance narrowbandwidth multi-color InAs/AlGaAs/GaAs quantum dot infrared photodetector," Jpn. J. Appl. Phys., vol. 44, pp. 6307-6311, 2005.

[4] S. D. Chen, Y. Y. Chen, and S. C. Lee, "Transverse-electric-field-enhanced response in InAs/AlGaAs/GaAs quantum-dot infrared photodetectors," Appl. Phys. Lett., vol. 86, pp. 2531041-2531043, 2005.

[5] S. Y. Lin, Y. J. Tsai, and S. C. Lee, "The effect of silicon dopant on the performance of InAs/GaAs quantum-dot infrared photodetectors," Jpn. J. Appl. Phys., vol. 42, pp. L167-L169, 2004.

[6] S. F. Tang, C. D. Chiang, P. K. Weng, Y. T. Gau, J. J. Luo, S. T. Yang, C. C. Shih, S. Y. Lin, and S. C. Lee, "High-temperature operation normal incident $256 \times 256 \mathrm{InAs}-\mathrm{GaAs}$ quantum-dot infrared photodetector focal plane array," IEEE Photon. Technol. Lett., vol. 18, no. 8, pp. 986-988, Apr. 15, 2006.

[7] T. Maier, H. Schneider, H. C. Liu, M. Walther, and P. Koidl, "Quantum-well infrared photodetector with voltage-switchable quadratic and linear response," Appl. Phys. Lett., vol. 88, pp. 051117-051119, 2006.

[8] P. Bhattacharya, X. H. Su, S. Chakrabarti, G. Ariyawansa, and A. G. U. Perera, "Characteristics of a tunneling quantum-dot infrared photodetector operating at room temperature," Appl. Phys. Lett., vol. 86, pp. 191061-191063, 2005.

[9] M. Z. Tidrow, X. Jiang, S. S. Li, and K. Bacher, "A four-color quantum well infrared photodetector," Appl. Phys. Lett., vol. 74, pp. 1335-1337, 1999.

[10] A. Lorke, R. J. Luyken, J. M. Garcia, and P. M. Petroff, "Growth and electronic properties of self-Organized quantum rings," Jpn. J. Appl. Phys., vol. 40, pp. 1857-1859, 2001.

[11] D. Granados and J. M. Garcia, "In(Ga)As self-assembled quantum ring formation by molecular beam epitaxy," Appl. Phys. Lett., vol. 82, pp. 2401-2403, 2003.

[12] F. Suarez, D. Granados, M. L. Dotor, and J. M. Garcia, "Laser devices with stacked layers of InGaAs/GaAs quantum rings," Nanotechnology, vol. 15, pp. S126-S130, 2004.

[13] P. Offermans, P. M. Koenraad, J. H. Wolter, D. Granados, J. M. Garcia V. M. Fomin, V. N. Gladilin, and J. T. Devreese, "Atomic-scale structure of self-assembled $\operatorname{In}(\mathrm{Ga})$ As quantum rings in GaAs," Appl. Phys. Lett., vol. 87, pp. 131902-131904, 2005.

[14] S. Y. Lin, Y. R. Tsai, and S. C. Lee, "Transport characteristics of InAs/ GaAs quantum-dot infrared photodetectors," Appl. Phys. Lett., vol. 83, pp. $752-754,2003$

[15] A. D. Stiff, S. Krishna, P. Bhattacharya, and S. W. Kennerly, "Normal-incidence, high-temperature, midinfrared, InAs-GaAs vertical quantum-dot infrared photodetector," IEEE J. Quantum Electron. vol. 37, no. 11, pp. 1412-1419, Nov. 2001.

[16] S. J. Xu, S. J. Chua, T. Mei, X. C. Wang, X. H. Zhang, G. Karunasiri, W J. Fan, C. H. Wang, J. Jiang, S. Wang, and X. G. Xie, "Characteristics of InGaAs quantum dot infrared photodetectors," Appl. Phys. Lett., vol. 73, pp. 3153-3155, 1998.

[17] S. Raghavan, P. Rotella, A. Stintz, B. Fuchs, S. Krishna, C. Morath, D. A. Cardimona, and S. W. Kennerly, "High-responsivity, normal-incidence long-wave infrared $(\lambda \sim 7.2 \mu \mathrm{m}) \mathrm{InAs} / \mathrm{In}_{0.15} \mathrm{Ga}_{0.85} \mathrm{As}$ dots-in-a-well detector," Appl. Phys. Lett., vol. 81, pp. 1369-1371, 2002.

[18] L. Jiang, S. S. Li, N. T. Yeh, J. I. Chyi, C. E. Ross, and K. S. Jones, " $\mathrm{In}_{0.6} \mathrm{Ga}_{0.4} \mathrm{As} / \mathrm{GaAs}$ quantum-dot infrared photodetector with operating temperature up to $260 \mathrm{~K}$," Appl. Phys. Lett., vol. 82, pp. 1986-1988, 2003. 